\title{
An introduction to neutrons for biology
}

\author{
Sophie Combet ${ }^{1,}$ * \\ ${ }^{1}$ Laboratoire Léon-Brillouin (LLB), UMR 12 CEA-CNRS, Université Paris-Saclay, CEA-Saclay, \\ 91191 Gif-sur-Yvette CEDEX, France
}

\begin{abstract}
The overlap of biology and neutron scattering remains a relatively narrow domain of research. This is partly due to the a priori maladjustment between real space problems and methods based on spatial and temporal correlations. In addition, some major assets of neutron scattering, such as isotopic substitution, can be tricky with biological molecules. More generally, a mutual lack of knowledge of the two concerned communities precluded potential rich interactions in early times. However, the situation changed to the point that, today, biology represents a substantial part of the research activity at neutron facilities. The purpose of this introduction is not to present one more overview of the subject of "neutron scattering" (excellent comprehensive articles are easily accessible to the interested readers $[1-4]$ ), but rather to facilitate the reading of the present book by introducing a few neutron scattering notions that may be useful for the community of biologists eventually less familiar with this technique.
\end{abstract}

\section{How can neutrons be a useful probe in biology?}

Biology is a wide field whose subjects of study are as diverse as populations, organisms, cells, or macromolecules. Actually, almost only biologists interested by biological macromolecules (proteins, nucleic acids, or lipid membranes constituting the living cells) or by issues at the molecular scale can be concerned by neutron scattering techniques.

A crucial issue in biology remains the knowledge of how atoms and molecules organize themselves and move to accomplish a biological function. The long-term "dream" is to observe macromolecules at work in vivo and to describe not only the positions of atoms but also the atomic motions and conformational changes that occur.

To probe structure, interactions, or dynamics of biomacromolecules, the "classical" techniques currently used by biologists are X-ray crystallography, nuclear magnetic resonance (NMR) spectroscopy, or the increasingly used cryo-electron microscopy. However, neutron scattering, as a complementary or even alternative technique, can prove to be essential to answer specific but relevant questions of biology.

In practice, the success of any technical approach depends on its insertion in modern biology through several parameters: high throughput, automatization, facilities to label the samples, perform the experiment, treat the data, etc. Today, neutron scattering is still "underused" in biology, where it occupies a kind of "niche". Such situation reduces its potential

\footnotetext{
* Corresponding author: sophie.combet@cea.fr
} 
importance and impact in the community of biologists. This is partly due to the a priori maladjustment between real space problems in biology and scattering methods, which are based on spatial and temporal correlations. More deeply, this under-utilization may be due to communication difficulties between physicists and biologists. For instance, the notion of "complexity" in biology drastically prevents or makes very difficult the practice of mathematical tools always present in physical models. There is therefore a "risk" for neutron users to move from biology issues to macromolecular physics.

This situation is also due to more "practical" reasons, such as difficulties of access, since neutron beams are produced only at nuclear reactors or spallation sources and thus are not accessible on a laboratory bench. The quantity and quality of sample necessary for a neutron experiment can sometimes be an obstacle in biology, although it depends on the beam intensity and the instrument used [5]. A special attention has indeed to be paid to the quality control of sample purity, monodispersity, and the monomeric state of the sample (e.g. by using SDS-PAGE, analytical NMR, SEC-DLS, or SEC-MALS) before but also all along an experiment, whose duration (from a few minutes to several days) can be tricky for most of biomolecules [5] (see also the book section written by Jeffries et al.). As such, the second generation of neutron spallation sources, like the European Spallation Source (ESS, Lund, Sweden) that should be operational in the next years, will open up new perspectives.

Like other neutron users, biologists can be driven by local contacts to perform a neutron experiment. As mentioned above, access to neutron experiments remains difficult since beam time is mainly accessible through experiment proposals evaluated by ad hoc scientific committees. Besides, there are many different home-made methods to reduce the data in neutron facilities that can make data treatment difficult but local contacts are usually available to provide help for this step.

Fortunately, recently, the rapid and huge development of neutron instruments, sample environments, tools for data treatment, and computer simulations [6,7] changed the situation to the point that nowadays biology represents a substantial part of the research activity at neutron facilities.

\section{The neutron scattering technique}

\subsection{Properties of neutrons}

Neutrons are nuclear particles discovered by J. Chadwick in 1932 (less than one century ago!) and were used the first time in 1946 by C. Shull as a tool for scattering experiments. Neutrons can be produced by two different ways (nuclear fission or spallation). Neutron scattering technique has since been developed to concern now all the aspects of condensed matter: physics, chemistry, materials, and so, as far as we are concerned, biology. Neutrons are an exceptional tool because they interact with atomic nuclei with very small energy, therefore without physical or chemical damage of the samples.

The energy of thermal neutrons is, by definition, that of room temperature, i.e. $\sim 25$ $\mathrm{meV}$, since they are thermalized at room temperature. Such domain of energy is often that of molecular motions (diffusion, rotation) and of chemical reactions. From this point of view, biology is an ideal domain to apply neutron scattering, as many biological processes take place also at room temperature. However, the large size of biomolecules, even of their secondary and tertiary structures, implies motions too slow to be accessible to neutrons, limiting substantially the potential dynamical studies.

Indeed, with typical wavelengths between 1 and $10 \AA$, neutrons cover ideally the $\mathrm{nm}$ and the ps space-time domain. Modern instruments succeed to extend this domain to two or 
three orders of magnitude, in the domain of hundreds of nm or several ns, but with a lower quality of the data and longer data acquisition times.

\subsection{Principles of neutron scattering}

Quantum mechanics associates any moving particle to a wave of wavelength $\lambda=\frac{h}{p}$ and energy $E=\hbar \omega$, where $h=2 \pi \hbar$ is the Planck constant and $p=m v$ is the momentum of a particle of mass $m$ moving with the velocity $v$.

Like in other scattering experiments, a neutron scattering experiment consists of sending a monochromatic beam of particles to a sample. Incident neutrons are defined by the momentum, thus by the wave vector $\vec{k}_{i}$, and energy $E_{i}$ :

$$
\begin{gathered}
\left\|\vec{k}_{i}\right\|=\frac{2 \pi}{\lambda_{i}} \\
E_{i}=\frac{\hbar^{2} k_{i}^{2}}{2 m}=\left(\frac{h^{2}}{2 m}\right)\left(\frac{1}{\lambda_{i}^{2}}\right)=\frac{1}{2} m v_{i}^{2}
\end{gathered}
$$

Neutrons interact with the nuclei of the sample through the nuclear interaction potential. They are scattered by the sample and measured in function of their wave vector $\vec{k}_{s}$ and energy $E_{s}$, providing information about the properties of the sample. $E$ :

The scattering vector is defined by the wave vector $\vec{Q}$ (Fig. 1) and the exchanged energy

$$
\begin{gathered}
\vec{Q}=\vec{k}_{i}-\vec{k}_{s} \\
E=\hbar \omega=E_{i}-E_{S}
\end{gathered}
$$
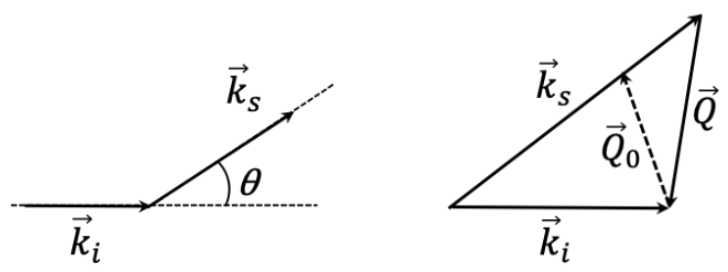

Fig. 1. Principle of a scattering experiment in the reciprocal space. Due to interaction with atoms of the sample, neutrons undergo a modification of their wave vector and energy. The case $\vec{Q}=\vec{Q}_{0}$ corresponds to elastic scattering, for which $\left\|\vec{k}_{i}\right\|=\left\|\vec{k}_{s}\right\|$ and $\omega=0$.

In the case of inelastic scattering $\left(\left\|\vec{k}_{i}\right\| \neq\left\|\vec{k}_{s}\right\|\right)$, we can define the modulus of the vector $\vec{Q}$ (Fig. 1) by:

$$
Q=\sqrt{k_{i}^{2}+k_{s}^{2}-2 k_{i} k_{s} \cos \theta} \quad \text { Eq. } 1
$$


In elastic scattering, i.e. without any energy exchange between the neutrons and the sample $\left(E_{i}=E_{s},\left\|\vec{k}_{i}\right\|=\left\|\vec{k}_{s}\right\|\right)$, we can define the modulus of the elastic scattering vector, noted $\vec{Q}_{0}$ (Fig. 1) by:

$$
Q_{0}=\frac{4 \pi}{\lambda} \sin (\theta / 2)
$$

where $\theta$ is the scattering angle. Note that $Q$ depends on the instrumental conditions $(\lambda, \theta)$, not on the sample.

Each incident neutron is described by a planar wave defined by a wave vector $\vec{k}$ that, when interacting with a single atom, is scattered isotropically in all directions, generating a spherical wave because the wavelength $\left(10^{-8} \mathrm{~cm}\right)$ is much larger than the size of an atomic nucleus (of the order of $\mathrm{fm}=10^{-13} \mathrm{~cm}$ ) (Fig. 2). The anisotropic nature of the scattering is due to the interference of multiple spherical waves centered in the different nuclei that constitute the sample.

Note that neutron scattering does not measure distances like a microscope would do. It measures correlation lengths and correlation times in the reciprocal space.

\subsection{Interferences}

Since the atomic nuclei are much smaller than the wavelengths used for neutron scattering, the nucleus acts as a point scatterer, resulting in isotropic scattering. For an incident plane wave described by the wave function $\Psi$, with unit amplitude, travelling in the positive $z$ direction with wavenumber $k_{i}=\left\|\vec{k}_{i}\right\|$, the scattered wave $\Psi^{\prime}$ is then spherical (Fig. 2 ), with an amplitude $\frac{b}{r}$ specific of the atomic nucleus, where $r$ is the distance from the nucleus (placed at $r=0$ ):

$$
\begin{gathered}
\Psi=\mathrm{e}^{i k_{i} z} \\
\Psi^{\prime}=-\frac{b}{r} \mathrm{e}^{i k_{S} r}
\end{gathered}
$$

The positive constant $b$ is the scattering length of a nucleus and is a measure of the "strength" of its interaction with the neutrons.

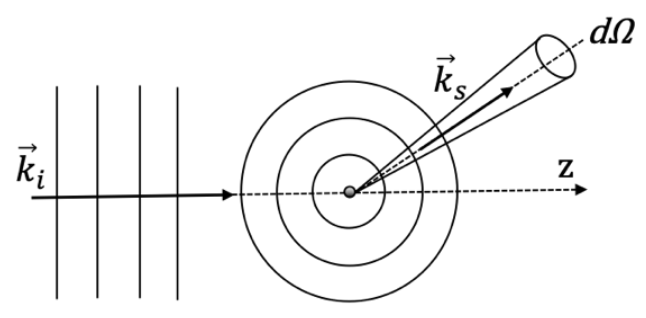

Fig. 2. Representation of the incident planar wave, with the wave number $\vec{k}_{i}$, scattered at a point scatterer into a spherical wave with the wave number $\vec{k}_{s}$. 
The incident neutron flux is equal to:

$$
\Phi=v\|\Psi\|^{2}=v
$$

The number of neutrons scattered by time unit through $d S$, the surface element (on a detector for instance) defined by the solid angle $d \Omega=\frac{d S}{r^{2}}$, can be calculated by:

$$
v d S\left\|\Psi^{\prime}\right\|^{2}=v d S \frac{b^{2}}{r^{2}}=v b^{2} d \Omega
$$

where $v$ is the neutron velocity and $\left\|\Psi^{\prime}\right\|^{2}$ is the probability of finding neutrons in $d S$.

The number of neutrons per solid angle is the differential scattering cross section $\frac{d \sigma}{d \Omega}$ and is calculated by the ratio of the scattered neutrons divided by the incident flux and the solid angle:

$$
\frac{d \sigma}{d \Omega}=\frac{v b^{2} d \Omega}{\Phi d \Omega}=b^{2}
$$

By integrating on the sphere around the sample (represented by a point scatterer), we find the differential section of a nucleus (the usual unit is barn, equal to $10^{-24} \mathrm{~cm}^{2}$ ):

$$
\sigma=4 \pi b^{2}
$$

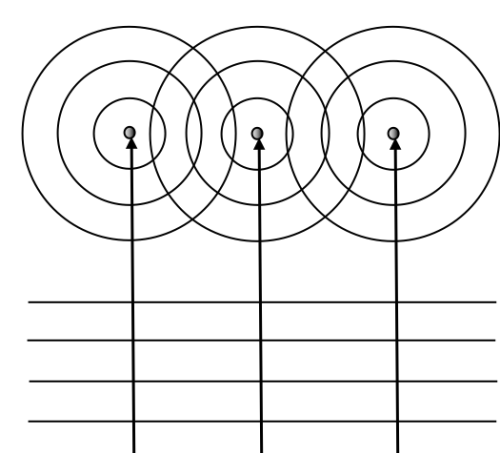

Fig. 3. Principle of a neutron scattering experiment showing interferences between three spherical waves generated by the interaction of the neutron planar wave with three point scatterers representing three nuclei in the sample.

In a neutron scattering experiment, the spherical waves, generated by the sample nuclei from the planar wave of each incident neutron, interfere with each other (Fig. 3). Some interferences between $i$ and $j$ nuclei are, either constructive, or destructive, therefore the spherical symmetry is lost and we observe oscillations. The differential scattering cross section, that makes an average in the ensemble, is written:

$$
\frac{d \sigma}{d \Omega}=\frac{1}{N}\left\langle\sum_{i \leq j} b_{i} b_{j} e^{\left(i \vec{Q} \cdot \vec{r}_{i j}\right.}\right\rangle \quad \mathbf{E q} \cdot 2
$$




\subsection{Coherent/incoherent scattering}

The term "coherence" we use for neutrons is not related to a property of the sample coherence (spatial correlation), nor to the neutron beam (which is rigorously incoherent).

In the interaction between a neutron and a nucleus, except the case of isotopes with null nuclear spin (e.g. C, O), there are two possibilities: the neutron couples with the nuclear spin of each atom (noted $I$ ), either parallel, or anti-parallel. Since the neutron has a spin equal to $\frac{1}{2}$, the spin of the ensemble, for each isotope, is: $I+\frac{1}{2}$ (parallel spins) or $\left|I-\frac{1}{2}\right|$ (anti-parallel spins) with the respective probabilities (weighting factors): $f_{+}=\frac{I+1}{2 I+1}$ and $f_{-}=\frac{I}{2 I+1}$.

Each interaction process corresponds to different scattering lengths, $b_{+}$and $b_{-}$, respectively. We can define the average and the standard deviation, that give, respectively, the "coherent" and "incoherent" scattering lengths:

$$
\begin{gathered}
b_{\text {coh }}=\bar{b}=f_{+} b_{+}+f_{-} b_{-} \\
b_{\text {inc }}=\sqrt{\overline{b^{2}}-(\bar{b})^{2}}
\end{gathered}
$$

The "coherent" scattering gives information on the spatial and temporal coherences between positions and motions of pairs of atoms nuclei in the sample. The coherent part of the scattered intensity depends therefore on the interaction between pairs of atoms and on collective excitations.

The "incoherent" scattering does not give any information about pair correlations as it depends only on individual nuclei and so gives information about individual (or self) correlations. Self-correlation techniques are able to probe dynamics of individual atoms, even in a concentrated sample.

\section{The "contrast variation" technique in biology}

Neutrons interact with the nuclei of atoms, not with electrons like X-rays do. Because the size of an atomic nucleus is several orders of magnitude smaller than the neutron wavelength, the scattering is isotropic and the scattering length $\left(b_{\text {nucleus }}\right)$, which corresponds to the atomic "scattering power", does not depend on the scattering angle. 


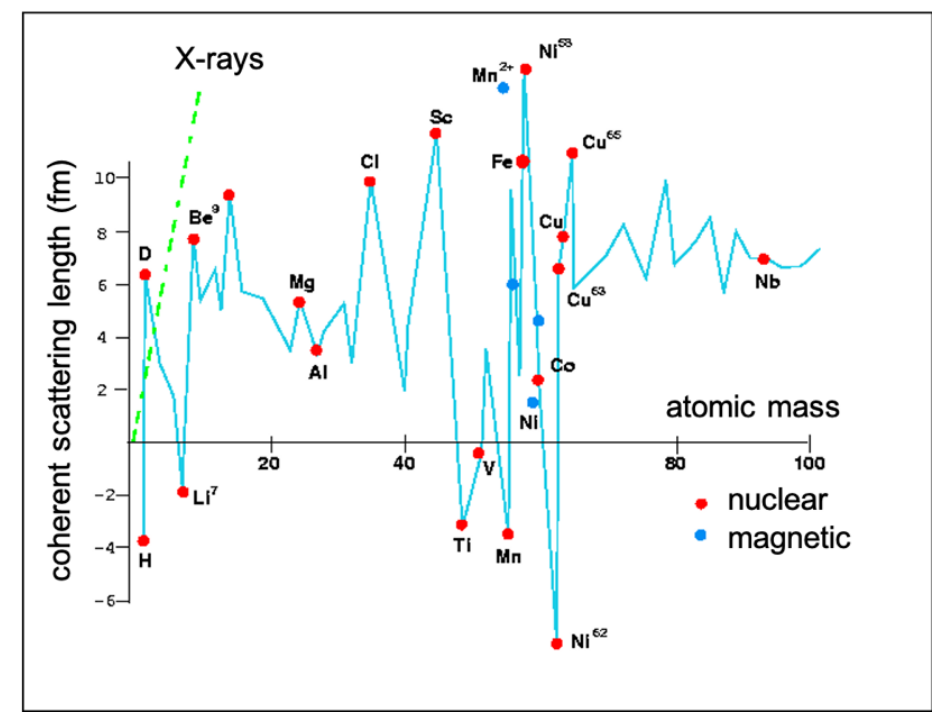

Fig. 4. Coherent scattering lengths of isotopes. Note that there is no relationship with their atomic numbers, in contrast to X-ray scattering lengths.

Contrary to X-ray scattering, the nuclear scattering length of atoms is independent of their atomic numbers (Fig. 4). Therefore, light and heavy elements have comparable scattering lengths. Moreover, different isotopes of a given element scatter neutrons in different ways. Some can exhibit very different scattering lengths and even with opposite signs (the negative sign of $b$ can be interpreted as the change of phase between the scattered and the incident waves).

In particular, this is the case for the coherent scattering length of hydrogen $\left(b_{H}=-3.74\right.$ $\mathrm{fm})$ and deuterium, which is positive and very large $\left(b_{D}=6.67 \mathrm{fm}\right)$, and close to that of other atoms found in biological samples (Fig. 5A). The large difference between the coherent scattering length of these two isotopes is very useful for structural studies of biomolecules, namely in techniques such as neutron diffraction, small-angle neutron scattering (SANS), specular neutron reflectometry (SNR), or neutron spin-echo spectrometry (NSE).

The difference between the scattering lengths, or the scattering length densities (which are calculated by dividing the added scattering lengths of atoms that constitute parts of the scattering entities by the occupied volume), allows the definition of a "contrast".

As mentioned above, in biology, as well as in organic compounds, the remarkably large difference between the coherent scattering length of hydrogen and that of other common atoms (deuterium, carbon, oxygen, nitrogen, phosphorous) allows large contrasts between parts of the sample, if one replaces selectively, hydrogen by deuterium. We have also to take into account the exchangeable $\mathrm{H}$ nuclei from the sample with the buffer used, that are those bound to $\mathrm{N}$ and $\mathrm{O}$ atoms (present in $\mathrm{NH}, \mathrm{NH} 2, \mathrm{OH}$, etc.), and called the "labile" protons, but not bound to $\mathrm{C}$ atom $(\mathrm{CH}, \mathrm{CH} 2, \mathrm{CH} 3$, etc.). See the book section of Jeffries et al. for more details.

Isotopic mixtures of $\mathrm{H}$ and $\mathrm{D}$ (for example, in hydrogenated and deuterated water) can therefore modulate the scattering length density of parts of a sample or of a solvent, erasing the contrast of such parts, thus enhancing the contrast of other parts. This technique, only possible with neutrons, is called "contrast matching" (Fig. 5). For instance, by mixing light and heavy water (or any other hydrogenous and deuterated solvents or buffers), the scattering length density of the solvent can be varied as desired. In the case of mixtures of compounds 
having different scattering lengths (hydrogenous and deuterated protein complexes, mixtures of hydrogenous and deuterated lipids, hydrogenous proteins in interaction with deuterated lipid membranes, hydrogenous proteins in the presence of a high concentration of deuterated crowding agents, etc.), it is therefore possible to mask either of the components of the compounds.

This is particularly important in biology where hydrogen is very present $(50 \%$ of all the atoms in most of biological macromolecules) and practically evenly distributed, which is a great advantage to make structural and dynamical studies using neutron scattering. Often, a neutron scattering measurement of a biological sample consists of measuring correlations between regions of the sample rich in hydrogen compared to other parts of the sample.

The notion of contrast must be applied differently at different scales, because a domain can be taken as homogeneous (thus, described by a single scattering length density) at a large scale, and heterogeneous at smaller scales, in which case the contrast must be defined with higher spatial resolution. Therefore, a large macromolecule can be defined by a single or by different scattering length densities at small or large $Q$-values, respectively. For example, amino acids constituting a protein can be distinguished or not, depending on the covered $Q$ range of the experiment.

A

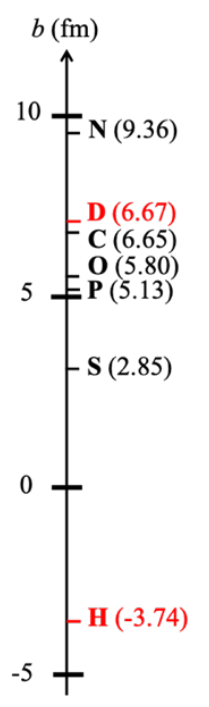

B

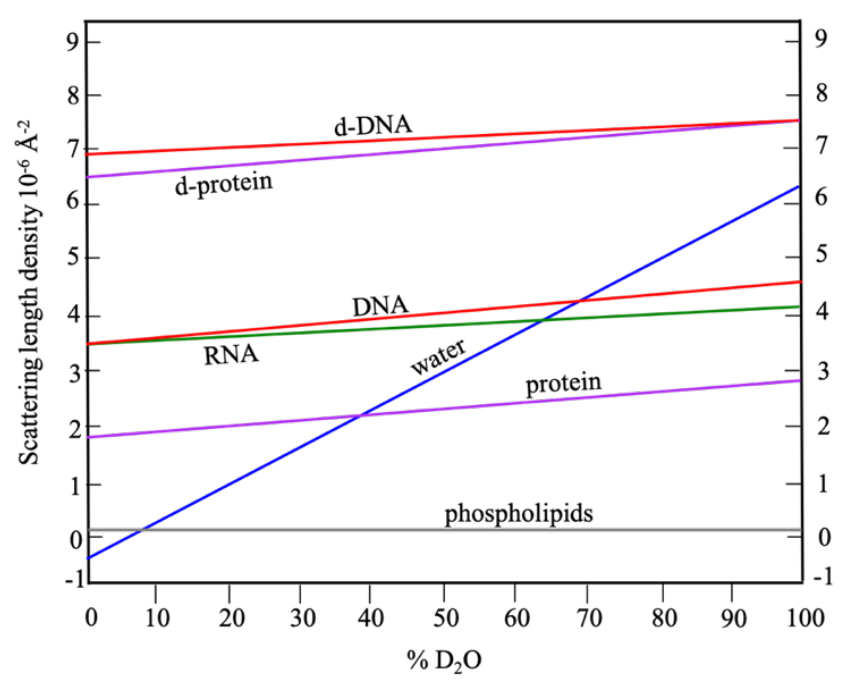

Fig. 5. (A) Coherent scattering lengths, in $\mathrm{fm}\left(1 \mathrm{fm}=10^{-13} \mathrm{~cm}\right)$ of the main atoms present in biological molecules. Note that the value of the hydrogen atom $\left({ }^{1} \mathrm{H}\right)$ is largely different from those of other atoms, in particular of the deuterium atom $\left({ }^{2} \mathrm{H}=\mathrm{D}\right)$, which allows high contrasts and is the basis of the "contrast variation" technique. (B) Scattering length densities of the natural and of a few fully deuterated biological molecules as a function of the isotopic composition of water. Lines are not horizontal because of labile hydrogen atoms, i.e. atoms of the solute that exchange with atoms of the solvent. This graph applies to water and, within good accuracy, to common buffers.

Some isotopes have also very different incoherent scattering cross sections. This is also the case of $\mathrm{H}$ and $\mathrm{D}\left(\sigma_{\text {inc }}(H) \simeq 80\right.$ barn compared to $\sigma_{\text {inc }}(D) \simeq 2$ barn $)$. Because of this large incoherent cross section, the motions of hydrogen atoms can be observed directly in quasielastic scattering, evaluated by time-of-flight spectroscopy (see 4.2). 


\section{What type of information does neutron scattering bring to biology?}

Several neutron scattering techniques are used in biology to study structure (crystallography [8], SANS (combined with simulations [9], SAXS [10,11], or NMR [12]), and reflectometry $[13,14]$ ) and dynamics $[15,16]$, which are presented in the present book. The reader is invited to refer to the following book sections to focus on:

- Neutron diffraction (“Opportunities and challenges in neutron crystallography” by N. R. Zaccai and N. Coquelle),

- Small-angle neutron scattering theory ("The basics of small-angle neutron scattering" by C. M. Jeffries, Z. Pietras, and D. I. Svergun); applications ("The power of SANS, combined with deuteration and contrast variation, for structural studies of functional and dynamic biomacromolecular systems in solution" by E. Mahieu, Z. Ibrahim, M. Moulin, M. Härtlein, B. Franzetti, A. Martel, and F. Gabel), and simulations ("Solution structure of macromolecules using small-angle neutron scattering and molecular simulations" by J. Bhatt),

- Neutron reflectometry theory ("An introduction to neutron reflectometry" by F. Cousin and G. Fadda) and applications ("Applications of neutron reflectometry in biology" by Y. Gerelli),

-Quasi-elastic and inelastic neutron scattering ("Probing the dynamics of biological matter by elastic, quasi-elastic, and inelastic neutron scattering” by G. Schirò).

Figure 6 compares the space-time domains covered by different neutron spectrometers compared to other techniques. It is worth noting that methods are complementary and cannot be compared in a simple way. This is because $i$ ) different probes interact with the sample in different ways and $i$ ) measured correlations correspond to individual, pair, or collective quantities. 


\section{JDN 24}

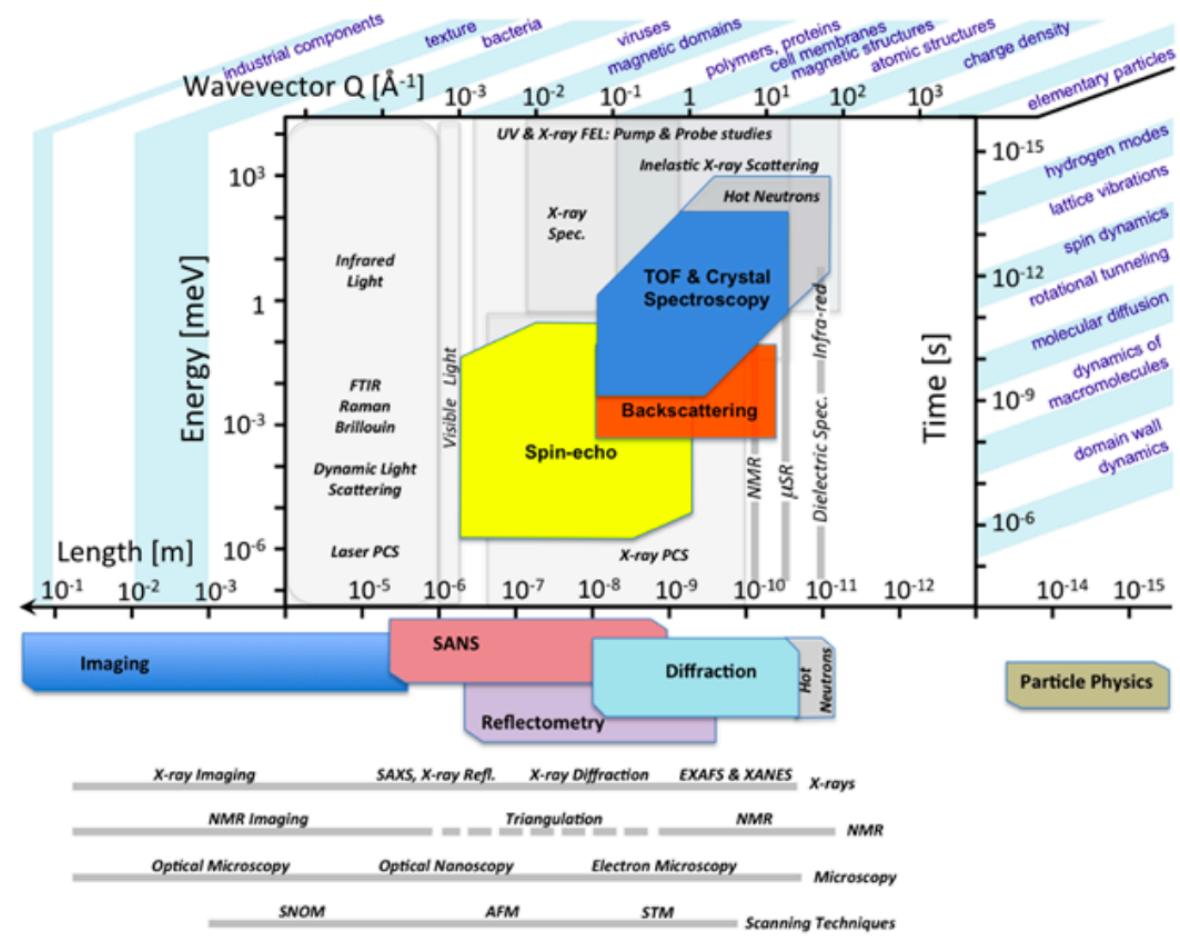

Fig. 6. Complementarity of inelastic neutron scattering, time-of-flight, backscattering, and neutron spin-echo with other spectroscopic techniques. The spatial $\left(r[\AA] \approx 2 \pi / Q\left[\AA^{-1}\right]\right)$ and temporal $(t[p s] \approx$ $0.658 / \hbar \omega[\mathrm{meV}])$ regions associated with the main physical phenomena potentially observable are shown. One can note that neutron scattering has the capability to simultaneously probe a significant region of this spatial and temporal map. Also, the spatial dependence of the correlation time is directly accessible. From [17] and the European Spallation Source (ESS) web site (https://europeanspallationsource.se/science-using-neutrons).

To give a flavor of the following book sections, we give below a succinct description of both structural and dynamical information neutron scattering can bring to biology, hoping that "appetite will come with eating".

\subsection{Structural information}

In structural studies (spatial correlations), incoherent scattering generates a constant background that adds to the coherent intensity, the only one containing information about spatial correlations in the sample. This is illustrated by the following equations. The general relation of neutron intensity can be written from Eq. 2 (see 2.3):

$$
S(\vec{Q}, t)=\sum_{i, j} b_{i}^{c o h} b_{j}^{c o h}\left\langle e^{\left[i \vec{Q} \cdot \vec{r}_{i}(0)\right]} e^{\left[-i \vec{Q}_{\vec{r}}(t)\right]}\right\rangle+\sum_{i}\left(b_{i}^{i n c}\right)^{2}\left\langle e^{\left[i \vec{Q} \cdot \vec{r}_{i}(0)\right]} e^{\left[-i \vec{Q} \cdot \vec{r}_{i}(t)\right]}\right\rangle
$$

When using the static approximation, we obtain: 


$$
S(\vec{Q})=\sum_{i, j} b_{i}^{c o h} b_{j}^{c o h} e^{\left[i \vec{Q} \cdot\left(\vec{r}_{i}-\vec{r}_{j}\right)\right]}+\sum_{i}\left(b_{i}^{i n c}\right)^{2}
$$

In a totally random sample, the coherent scattered intensity is null. However, in biological samples, like in condensed matter in general, correlations are always present, at least due to steric effects or to compressibility in the case of fluids.

The extension of spatial correlations goes from interatomic distances (e.g. atoms of a molecule) to infinity, in a perfect crystal. A particular neutron technique can measure correlations within a relatively small spatial range, reason why different instruments are necessary to evaluate coherences at different scales.

Elastic scattering gives access to the structural properties of matter, i.e. the way atoms, molecules or complex structures organize themselves and interact in space. Information is always derived from spatial correlations between sites or spatial domains. The size of such domains goes from 0.1 to $1,000 \mathrm{~nm}$.

High spatial resolution is mostly used to determine crystalline structures by diffraction. Most of the time, neutron diffraction complements X-ray diffraction, in particular to determine positions of hydrogen atoms or protons $\left(\mathrm{H}^{+}\right)$, which interact too weakly with electromagnetic radiation. The basic principles of neutron diffraction are similar to those of $\mathrm{X}$-rays. In certain directions, the periodicity of the lattice of a crystal induces strong constructive interferences resulting in the observation of Bragg peaks (for more details, especially on diffraction theory, refer to the book section written by Zaccai and Coquelle).

With a much lower spatial resolution, small-angle neutron scattering (SANS) probes global sizes and shapes of macromolecules in solution as well as their mutual interactions. Obtained results are very reliable because they correspond to statistical averages over an extremely large number of scattering entities, essentially, all those contained in a sample volume of the order of $100 \mathrm{~mm}^{3}$. Cryo-microscopy can give equivalent information but statistics refers only to a few molecules and the necessary preliminary preparation of the sample can influence the observations. On the other hand, random inhomogeneity is averaged and is therefore not observed in reciprocal space.

Measuring with high resolution the small $Q$-domain, SANS of biomolecules in solution can often be decomposed into two independent terms. One of them, dominating the large $Q$ domain, gives information about size, shape, and surface area of the molecules, while scattering at lower angles gives information about mutual interactions. In this case, scattering is the most precise technique allowing the evaluation of interactions, thus of the effective potentials that define large-scale interactions. Within simple, often valid, assumptions, the total scattered intensity is the product of two terms, form and structure factors describing molecular shape and interactions, respectively.

Whenever reliable, extrapolation to zero angle of scattered intensity yields information about thermodynamic quantities such as molecular mass or osmotic pressure. Even less accurate SANS data can give information about global quantities such as radius of gyration $\left(R_{g}\right)$ of an aggregate or a complex, or about the area of interfaces. Indeed, at large $Q$-values, SANS probes correlations at small length scales, i.e. intra-particle correlations, while at the smallest $Q$-values, large correlation lengths characterizing inter-particle correlations are the main contribution to the scattered intensity (Fig. 7). 


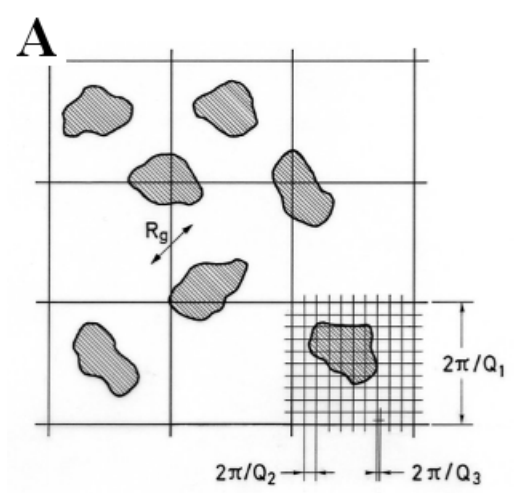

B

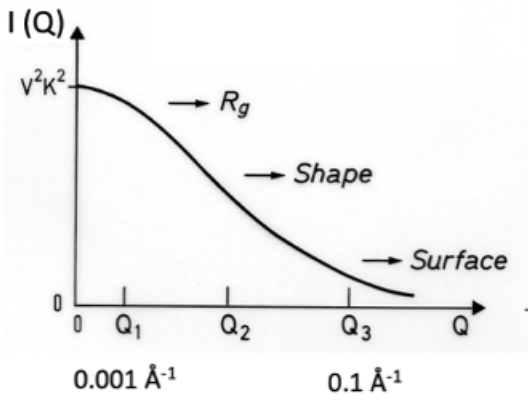

Fig. 7. (A) Schematic representation of a two-dimensional sample, showing different degrees of spatial resolution as a function of $Q$ and $(\mathbf{B})$ the corresponding regions of the form factor $(I(Q)=P(Q))$ curve. From [1].

In real space, the product of form and structure factors corresponds to the convolution of the self-correlation of the particles with the pair correlation function that describes the spatial arrangements of the particles in the solution.

More details on SANS theory, applications, and simulations can be found in the book sections written by Jeffries et al., Mahieu et al., and Bhatt.

Scattering principles of specular neutron reflectometry (SNR) are the same as for SANS but applied to surfaces, instead of volumes. SNR consists on sending a collimated neutron beam onto a surface with a certain incidence angle and measuring the reflectivity of the sample as a function of the vector scattering, noted $Q_{z}$, in the direction normal to the surface, and with an amplitude equal to:

$$
Q_{z}=\frac{4 \pi}{\lambda} \sin (\theta)
$$

where $\lambda$ is the neutron wavelength and $\theta$ the incidence angle (for more details, refer to the book section written by Cousin and Fadda). The reflectivity curve obtained makes it possible to determine the thickness and the scattering length density of each layer of the sample. This is called "specular" reflectivity (like a mirror) as opposed to the "grazing-incidence" (GISANS) SANS technique which provides information in the plane area.

\subsection{Dynamical information}

Neutron scattering uses "thermal neutron" particles that have been thermalized by inelastic exchanges with a thermal bath, either water at room temperature, or liquid hydrogen at $20 \mathrm{~K}$. The resulting kinetic energy of neutrons that interact with matter is similar to that of intermolecular bonds, thermally activated processes, or collective excitations, which are associated to energies smaller than a few tens of meV. Therefore, the energy domain probed by neutrons is very different from that of X-rays or light spectroscopy, for which the characteristic energy of the photons is one million or one thousand times larger, respectively.

Additionally, the interaction with the only atomic nuclei (we exclude magnetic interactions) makes the theory relatively simple because describing only atomic 
displacements (structural information) and vibrational and collective motions (dynamical information).

As pointed out above, a specific advantage of neutron scattering is the existence of two contributions to the scattered intensity, coherent and incoherent, which can be tuned often by isotopic substitutions that, within good approximation, do not modify physical or chemical properties of the samples. In practice, this remark applies almost exclusively to hydrogen and deuterium atoms, often present in biomolecules and hydration water.

While, in structural studies, the incoherent scattering represents a simple $Q$ independent background without information about pair correlations, in dynamic studies, it gives information about individual motions of atoms. This property is almost unique in scattering techniques, for which information about individual motions can be obtained but only indirectly from measurements of mutual diffusion coupled with appropriate models (for example, in dynamic light scattering).

However, in practice, measurements of individual motions are possible only for hydrogen atoms. This is because the total coherent scattering intensity is proportional to $N^{2}$ while the incoherent part is proportional to $N$, where $N$ is the number of nuclei in a sample. Only the very large incoherent cross section of hydrogen, typically two orders of magnitude larger than all other nuclei other nuclei found in biological samples $\left(\sigma_{\text {inc }}(H) \simeq 80\right.$ barn compared to $\sigma_{\text {inc }}(C, O, N, D) \simeq 0-2$ barn) can compensate for this difference.

Therefore, in practice, evaluations of individual dynamics are almost exclusively achieved through measurements of motions of hydrogen atoms.

In biology, atomic fluctuations on the ps-ns timescale are of particular interest because they result from and reflect the forces that structure biological macromolecules, including atomic motions and molecular flexibility associated with biological activity.

Studies of dynamics are however not as numerous as structural investigations. Beyond the fact that there are fewer problems related to microscopic dynamics, neutron scattering has several limitations both at the level of the samples and of the covered dynamical range.

Without going into details of each technique (for that, the reader can refer to the book section written by Schirò), we can briefly describe the main classes of techniques and instruments currently used.

\subsubsection{Quasi-elastic neutron scattering}

Quasi-elastic neutron scattering (QENS), performed in time-of-flight (TOF) spectrometers, measures dynamical processes in the domain 1-100 ps with good accuracy. Through this technique, it is possible to discriminate between rotational and translational motions. It has been used in many studies of dynamics of water in hydrated molecules giving information about self-diffusion, rotational motions, jumps between sites, hydrogen bond lifetime, etc. It probes similar motions in biomolecules, as far as associated relaxation times are not too long as compared to the instrumental resolution, which is of the order of tens of $\mu \mathrm{eV}$.

Given the characteristic space-time domain better covered by neutron scattering, the majority of QENS studies concerns motions at molecular scale, meaning that collective motions (cf. 4.2.3) often associated to biological functions only indirectly can be taken into account. This is the case of the kinetics of protein folding, which is too long to fit in the experimental window. Instead, dynamical properties of stable refolding intermediates can be measured in order to be compared with those of the native state.

This technique allows a direct measurement of the van Hove function $G_{s e l f}(r, t)$ of the hydrogen atoms present in the sample (the following being related to isotropic systems, we substitute $r$ to $\vec{r})$. $G_{\text {self }}(r, t)$ is the probability of finding a hydrogen atom at point $r$ and at time $t$ assuming that the same atom was at the origin at time $t=0$. 


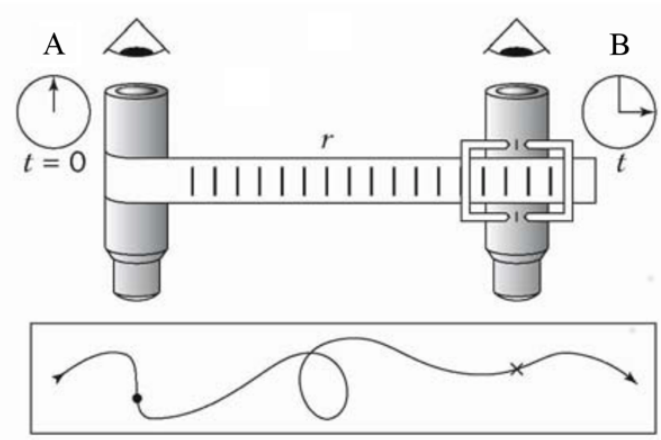

Fig. 8. A "fly correloscope". From [2].

To illustrate the meaning of $G_{\text {self }}(r, t)$ with an image, R. Scherm and B. Fåk [2] suggested the image of a "fly correloscope", constituted by two viewfinders A and B connected by a graduated ruler and a chronometer, to illustrate how to measure the probability of having a fly at $(\vec{r}, t)$ if we have seen the same fly at $(0,0)$ (Fig. 8).

Figure 9 depicts schematically the shape of $G_{s e l f}(r, t)$ in the case of a simple diffusion process with spherical symmetry.

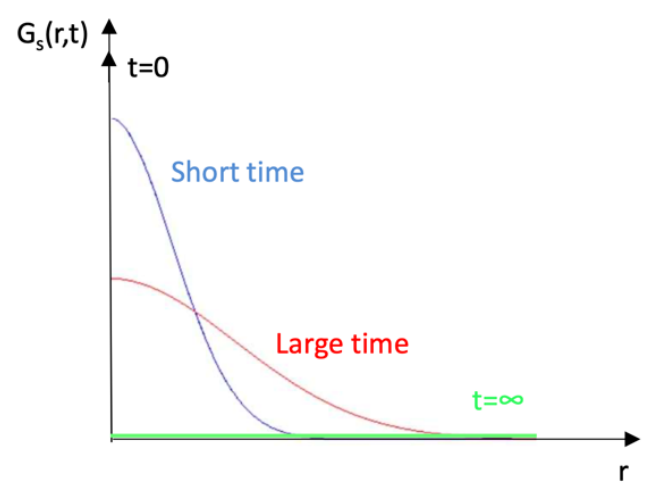

Fig. 9. Shape of $G_{\text {self }}(r, t)$ as a function of time.

At $t=0$, the diffusing particle is at the origin. With increasing time, it can be found in a larger region and, at infinite time, it can be anywhere in the sample. Obviously, all these bell-shaped functions have the same integral, equal to 1 .

In practice, the measurement can evaluate diffusive motions of large particles containing hydrogen atoms, as far as internal motions of hydrogen atoms take place at a different time scale. In case of rotational motions, the particle remains confined in a limited region of the space, meaning that $G_{\text {self }}(r, \infty)=$ constant $\neq 0$.

As we have seen above, about the structure factor, neutron scattering analyses correlations in reciprocal space, for which the variable reciprocal of the real space $r$ is the momentum exchange $Q$. Similarly, concerning dynamics, many instruments measure the energy exchange, $\Delta E=\hbar \omega$, between neutrons and nuclei, $\omega$ being the conjugate variable of time $t$. We illustrate below the relationships between the van Hove function and the spectra measured by neutron scattering: 


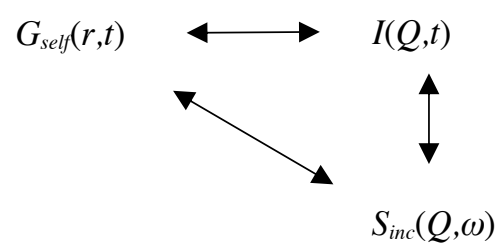

The index self refers to individual dynamics, which, most of the time (but not always) is measured by the incoherent part of the scattered intensity, $\operatorname{Sinc}(Q, \omega)$. Arrows represent space and time Fourier transforms. $I(Q, t)$ and $S(Q, \omega)$ are the intermediate and the dynamical scattering functions, respectively:

$$
S(\vec{Q}, \omega)=\frac{1}{h} \iint G(\vec{r}, \mathrm{t}) \exp [i(\vec{Q} \cdot \vec{r}-\omega t)] d \vec{r} d t
$$

Majority of spectrometers measure $S(Q, \omega)$. This is the case of different types of TOF and backscattering instruments. Typical energy resolutions are 50 and $0.1 \mu \mathrm{eV}$ (FWHM), respectively. Based on a different principle, spin-echo instruments measure with a high energy resolution $I(Q, t)$, i.e. relaxation times up to $10 \mathrm{~ns}$ (we remind that $t[p s] \approx 0.658 / \hbar \omega$ $[\mathrm{meV}])$, typical of the dynamics of large molecules or aggregates.

\subsubsection{Inelastic neutron scattering}

Inelastic neutron scattering (INS) probes atomic and molecular vibrational modes, namely the vibrational density of states related to thermodynamic properties and phase transitions. They are mostly in the intermolecular energy domain below $100 \mathrm{meV}$.

They are measured in TOF spectrometers, often with low energy resolution in order to reach high energy exchange at moderate $Q$ values.

Actually, as it can be seen in Figure 10, there is a strong interdependence between $Q$ and $\omega$, because of the small energy of neutrons. The neutron dispersion curve, that follows Eq. 1 (see 2.2), relates $Q$ and $\omega$ for given incident neutron wavelength $\lambda=2 \pi / k_{l}$ and scattering angle $\theta$. 


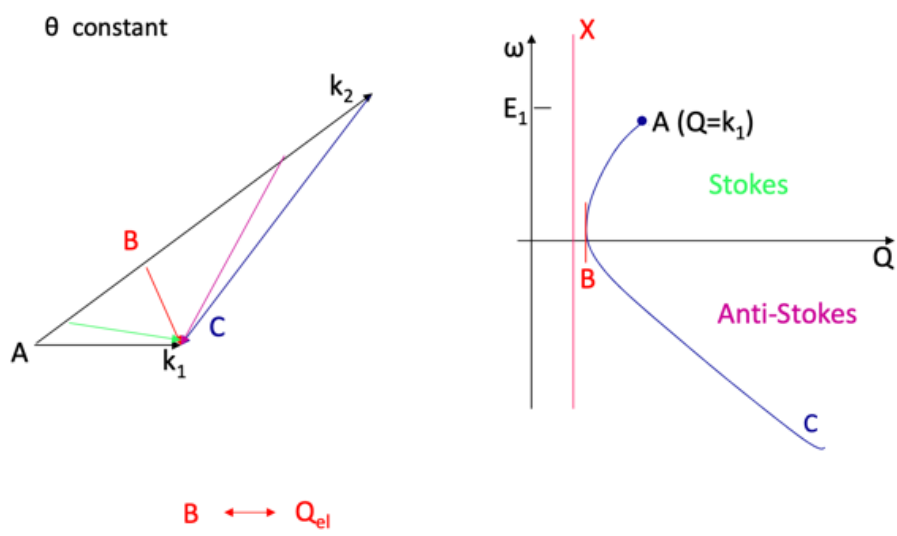

Fig. 10. The neutron dispersion curve relying the momentum $Q$ and the energy $\omega$, for a given wavelength $\lambda$ and a scattering angle $\theta$.

On the same scale, the equivalent curve for X-rays or light scattering is reduced to a vertical line because the incident energy is much higher than the energy exchanges probed by neutrons.

One consequence of the neutron dispersion is the small extension of the Stokes side (neutron energy loss) of the spectra. Therefore, analysis is done on the anti-Stokes side (neutron energy gain) despite the lower intensity, particularly at low temperature.

Still, INS is a very useful probe that, in biology, often complements light scattering in the difficult domain of far infra-red absorption (typically up to a few $\mathrm{THz}$, with $1 \mathrm{THz}=32$ $\mathrm{cm}^{-1}$ ) where intermolecular contributions dominate.

\subsubsection{Collective motions}

More rarely in biology, INS can probe collective excitations, namely propagative excitations and acoustic modes (sound waves). Equivalent of Brillouin light scattering in a domain of frequency three orders of magnitude larger ( $\mathrm{THz}$ instead of $\mathrm{GHz}$ ) the experiments evaluate the energy dependence of the coherent part of the scattering function, $S_{c o h}(Q, \omega)$ in the hydrodynamic limit, $i . e$. at small $Q$-values. Technically, experiments are difficult because only a narrow domain of $Q$ and wavelength is available and the scattering intensity is always weak. This is because typical values of sound velocity imply using short wavelength neutrons, hardly compatible with small-angle scattering.

Today, high resolution X-ray inelastic scattering is the main method in this domain of research.

\subsubsection{Elastic neutron scattering}

Strictly speaking, elastic scattering is solely due to immobile atoms or to local motions taking place at scales relatively small. Examples of the latter are intramolecular rotations of methyl groups or diffusion under high confinement.

The eventual $Q$-dependence of the intensity of the elastic line gives information about the geometry of the confined volume where motions, namely rotations, take place. An important quantity often evaluated from the elastic component is the elastic incoherent structure factor (EISF) that gives information about the size of a domain where confined 
motions take place. Therefore, although indirectly, it gives structural information about the sample.

However, in case of slow diffusion, more generally in case of long relaxation times, the instrumental resolution is not good enough to discriminate slow motions from real immobility. In practice, what is measured like "elastic" scattering, does include motions associated to long relaxation times. In elastic scans, one measures the intensity scattered inside a dynamic window generally centered at $\omega=0$. In general, its width is the instrumental resolution, eventually larger. Measurements are performed in function of an external parameter, normally the temperature.

In the case of a sample in which all atoms are immobile, one will observe a regular decrease of the "elastic" intensity due to the Debye-Waller factor, which describes atomic delocalizations. With increasing temperature, part of the intensity is scattered inelastically, reducing the intensity scattered elastically. Actually, beyond this trivial effect, one looks for kinks or discontinuities of the elastically scattered intensity plotted against temperature. They correspond to dynamic changes, such as onset of dynamic processes, glass or phase transitions.

It is worth noting that the most frequent dynamics of biomolecules is associated to relaxation times larger than $1 \mathrm{~ns}$, which are too large to be studied in TOF spectrometers. Therefore, spin echo instruments and elastic scans, often performed in backscattering instruments, are currently used.

Many other techniques are possible with neutrons in biology that are not described in the present book (e.g. imaging). We did not give also technical details on neutron sources and instruments (for that purpose, refer to [2,4]).

Nevertheless, we hope that we convinced the reader that neutron techniques are useful probes in many cases in biology and that this introduction encourages to go further, which is also the propose of the following chapters of this book, that we invite to discover.

\section{References}

1. J. Teixeira, Introduction to small angle neutron scattering applied to colloidal science, 1992, Struct. Dyn. Supramol. Aggregates Strongly Interact. Colloids, NATO ASI S, p. 635.

2. R. Scherm, B. Fåk, 1993, Neutron Synchrotron Condens. Matter Stud., Springer-V, Les Ulis, p. 113.

3. J. Schweizer, 2005, Neutrons Biol., Journal de Physique IV.

4. T. Ederth, Neutrons for scattering: What they are, where to get them, and how to deal with them, 2018, JDN 23 - French-Swedish Winterschool Neutron Scatt. Appl. to Soft Matter, EPJ Web of Conferences.

5. C.M. Jeffries et al., Nat. Protoc. 11, 2122 (2016)

6. D. Franke et al., J. Appl. Crystallogr. 50, 1212 (2017).

7. J. Trewhella et al., Acta Crystallogr. Sect. D Struct. Biol. 73, 710 (2017)

8. M.T. Banco et al., Proc. Natl. Acad. Sci. U. S. A. 113, 13756 (2016)

9. R. Dos Santos Morais et al., Biophys. J. 115, 1231 (2018)

10. F. Spinozzi et al., Langmuir 33, 2248 (2017)

11. M. Chevreuil et al., Nat. Commun. 9, 3071 (2018)

12. A. Lapinaite et al., Nature. 502, 519 (2013)

13. A. Martel et al., J. Am. Chem. Soc. 139, 137 (2017)

14. S. Combet et al., Biochim. Biophys. Acta - Biomembr. 1861, 514 (2019)

15. G. Schirò and M. Weik, J. Phys. Condens. Matter. 31, 463002 (2019)

16. S. Combet and J.-M. Zanotti , Phys. Chem. Chem. Phys. 14, 4927 (2012) 
17. Q. Berrod et al., 2018, Inelastic and quasi-elastic neutron scattering. Application to soft matter. French-Swedish Winterschool Neutron Scatt. Appl. to Soft Matter, EPJ Web of Conferences. 